\title{
The Risk of Post-operative Complications in Super-Super Obesity Compared to Super Obesity in Accredited Bariatric Surgery Centers
}

\author{
K. Hope Wilkinson ${ }^{1} \cdot$ Melissa Helm ${ }^{1} \cdot$ Kathleen Lak $^{1} \cdot$ Rana M. Higgins $^{1} \cdot$ Jon C. Gould $^{1} \cdot$ Tammy L. Kindel $^{1,2}$ (D)
}

Published online: 27 May 2019

(C) Springer Science+Business Media, LLC, part of Springer Nature 2019

\begin{abstract}
Background The prevalence of super obesity $\left(\mathrm{SO}, \mathrm{BMI}>50.0 \mathrm{~kg} / \mathrm{m}^{2}\right.$ ) and super-super obesity $\left(\mathrm{SSO}, \mathrm{BMI}>60 \mathrm{~kg} / \mathrm{m}^{2}\right)$ is increasing. Current data are limited and discrepant on the relationship between SSO and post-bariatric surgery complication risk. We hypothesized there would be increased complications for both laparoscopic Roux-en-Y gastric bypass (LRYGB) and sleeve gastrectomy (LSG) in SSO compared to SO, but the relative risk (RR) would support the use of LSG in SSO patients. Methods Metabolic and Bariatric Surgery Accreditation and Quality Improvement 2016 data were queried for SO and SSO patients undergoing LRYGB or LSG. Thirty-day post-operative complications were calculated. Univariate analyses were performed with a $\chi^{2}$ or Student's $t$ test. Comparisons between multiple groups were performed using a one-way ANOVA. Statistical significance was defined as $p<0.05$.

Results A total of 5723 patients with SSO and 24,940 with SO were included for analysis. Patients with SSO had more comorbidities. Patients with SSO had a higher likelihood of complications compared to SO patients $(15.2 \%$ vs $12.6 \%, p<0.0005)$. SSO patients, and specifically SSO RYGB, were significantly more likely to experience an unplanned intubation, prolonged ventilation, and unplanned ICU admission. Compared to SO LRYGB, the RR for complications in SSO LRYGB and LGS were 1.19 and 0.76 respectively $(p<0.0005)$.

Discussion We found SSO patients had increased 30-day post-operative complications after both LRYGB and LSG compared to SO patients. LSG may be the preferred procedure for this high-risk population.
\end{abstract}

Keywords Super obesity $\cdot$ Super-super obesity $\cdot$ Sleeve gastrectomy $\cdot$ Gastric bypass $\cdot$ Complications

\section{Introduction}

In the USA, 39\% of the population has the disease of obesity, representing more than 93 million US adults [1]. Super obesity (SO) is defined as body mass index (BMI) $\geq 50 \mathrm{~kg} / \mathrm{m}^{2}$ and super-super obesity (SSO) is defined as a BMI $\geq 60 \mathrm{~kg} / \mathrm{m}^{2}$. In 2010 , the prevalence of super obesity in the USA was $0.55 \%$, a $120 \%$ increase from the year 2000 [2]. In 2012, 24\% of all

Electronic supplementary material The online version of this article (https://doi.org/10.1007/s11695-019-03942-0) contains supplementary material, which is available to authorized users.

Tammy L. Kindel

tkindel@mcw.edu

1 Department of Surgery, Medical College of Wisconsin, 8900 W. Doyne Avenue, Milwaukee, WI 53226, USA

2 Department of Surgery, Medical College of Wisconsin, $8700 \mathrm{~W}$. Watertown Plank Road, Milwaukee, WI 53226, USA bariatric patients undergoing Roux-en- $\mathrm{Y}$ gastric bypass (RYGB) at accredited bariatric centers were SO and 7.6\% were SSO [3]. While there is an increasing prevalence of SSO patients undergoing bariatric surgical care [4], there is an under-representation of sufficiently powered studies looking at the incidence of peri-operative complications and if a specific procedure is preferred in the SSO population.

At our bariatric center, we have a preference to offer laparoscopic sleeve gastrectomy (LSG) to patients with SSO due to concerns about a significant step-up risk in peri-operative complications for patients with a BMI $\geq 60 \mathrm{~kg} / \mathrm{m}^{2}$ who undergo laparoscopic RYGB (LRYGB). However, the current literature does not show a consistent relationship between increased BMI and complication risk. Database analysis using National Surgical Quality Improvement Program (NSQIP) data from 2005 to 2008 and the Bariatric Outcomes Longitudinal Database (BOLD) registry between 2007 and 2009 reported an increased risk of complications in patients with $\mathrm{SSO}[3,5$, 6]. From the BOLD database, SO patients had an increased 
odds ratio of serious adverse events after gastric bypass of 1.25 (95\% CI 1.02-1.53) and SSO patients had a significantly increased odds ratio of 1.91 (95\% CI, 1.47-2.50) [3]. In the NSQIP-based risk calculator, the odds ratio for morbidity was 1.45 for patients with a BMI $>60 \mathrm{~kg} / \mathrm{m}^{2}$ compared to patients with a BMI 45 to $60 \mathrm{~kg} / \mathrm{m}^{2}$ for any bariatric procedure [5]. More recent studies from the Scandinavian Obesity Surgery Registry (SOSR) database and the Michigan Bariatric Surgery Collaborative did not find pre-operative BMI to be predictive of post-operative complications $[7,8]$.

This study was designed to use a national database, the American College of Surgeons Metabolic and Bariatric Surgery Accreditation and Quality Improvement (MBSAQIP) Data Registry, to determine if peri-operative complications increase and to a similar degree for both LSG and LRYGB when comparing SO to SSO patients. We hypothesized there would be an increase in complications for both procedures comparing $\mathrm{SSO}$ to $\mathrm{SO}$, but the relative risk of increase would be greater for LRYGB than LSG supporting the use of LSG specifically in SSO patients.

\section{Methods}

The MBSAQIP Data Registry was queried from 2016, containing 186,722 cases from 791 sites, and filtered for SO and SSO patients. The MBSAQIP captures the metabolic and bariatric cases performed in the USA and Canada at MBSAQIP participant centers. Data is entered by a certified metabolic and bariatric clinical reviewer. As the MBSAQIP gathers several different pre-operative BMIs per patient, we selected the patient's BMI closest to surgery for categorization into $\mathrm{SO}$ (BMI 50.00-60.00 kg/m²) or SSO (BMI 60.01-69.99 kg/m²).

We included patients whose primary procedure CPT code was 43,659 and 43,775 for LSG and 43,644 and 43,645 for LRYGB. We excluded cases which were coded as revisions/ conversions or involved concomitant removal of a gastric band. We also excluded patients who underwent the following concurrent procedures by CPT code: cholecystectomy, ventral/incisional hernia repair, mesh placement, complex wound closures or fat grafting, appendectomy, foreskin repositioning, tubal ligation, hysterectomy, IUD placement, lysis of spinal canal tissue, inferior vena cava (IVC) filter placement, colonoscopy, small intestinal endoscopy, uteroneocystostomy, cystoscopy, and hysteroscopy. Patients with an American Society of Anesthesiologists (ASA) class of 5 were excluded.

The following patient demographics were recorded for data analysis: age, gender, BMI, ASA class, race, smoking status, and functional status, as well as the presence of co-morbid conditions including chronic obstructive pulmonary disease (COPD), obstructive sleep apnea, gastroesophageal reflux disease, coronary artery disease, hypertension, hyperlipidemia, chronic kidney disease, and end-stage renal disease.

A variety of post-operative complications were collected including superficial incisional surgical site infection (SSI), deep incisional SSI, organ space SSI, wound disruption, pneumonia, re-intubation, pulmonary embolism, prolonged ventilation requirement $(>48 \mathrm{~h})$, urinary tract infection, cerebral vascular accident, cardiac arrest requiring cardiopulmonary resuscitation, myocardial infarction (MI), transfusion within 72 h of surgery, venous thrombosis, Clostridium difficile infection, post-operative sepsis, septic shock, coma, intensive care unit (ICU) admission, re-operation within 30 days, readmission within 30 days, emergency department (ED) visits without an associated re-admission, and death.

Descriptive statistics of categorical variables were calculated as totals and percentages and compared with a $\chi^{2}$ test. Continuous variables were compared using an independent Student's $t$ test. Aggregate complication rates were compared using one-way ANOVA. Relative risks were calculated using SO LRYGB as the control group. Logistic regression modeling, univariate and multivariate, was applied to selected comorbidities including obesity status OR BMI, and sex, age, diabetes mellitus, limited ambulation, hypertension, and sleep apnea, to evaluate for attributable differences in complications independent of obesity status presented as odds ratios (OR) and $95 \%$ confidence intervals (CI). Statistical analysis was performed using IBM SPSS Statistics version 24 (Armonk, NY: IBM Corp). Statistical significance was defined as $p<0.05$.

\section{Results}

A total of 186,772 bariatric operations were recorded in the (MBSAQIP) Data Registry for the year 2016. After exclusion criteria were applied, there were 30,663 patients remaining for analysis. Totaling both surgical procedures, there were 5723 patients with SSO. For SSO patients, $31.7 \%$ underwent LRYGB ( $n=1816)$ and $68.3 \%$ underwent LSG $(n=3907)$. With a very similar case distribution, 24,940 patients were classified as SO with $31.4 \%$ of patients undergoing LRYGB ( $n=7820)$ and $68.6 \%$ undergoing LSG $(n=17,120)$. Patients with SSO had statistically significantly longer procedures times $(92.7 \pm 51.0$ vs. $89.7 \pm 49.0 \mathrm{~min}, p<0.0001)$ and longer lengths of stay than SO patients $(1.88 \pm 1.4$ vs. $1.76 \pm 1.3$ days, $p<0.0001)$. Patients with SSO were more likely to have comorbidities of limited ambulation, hypertension, vein thrombosis, venous stasis, therapeutic anticoagulation, diabetes mellitus, partially or fully dependent, COPD, oxygen dependent, pulmonary embolism, obstructive sleep apnea, pre-op IVC filter and categorized as an ASA class 3 or 4 (Table 1). Patients with SSO had a higher likelihood of complications, with $15.2 \%$ of patients with SSO experiencing a complication 
Table 1 Demographics and presurgical co-morbidities of super obese (BMI $50-60 \mathrm{~kg} / \mathrm{m}^{2}$ ) compared to super-super obese patients (BMI 60.1-69.9 kg/m²) undergoing both Roux-en-Y gastric bypass and sleeve gastrectomy

\begin{tabular}{|c|c|c|c|}
\hline & $\begin{array}{l}\text { Super obese } \\
(n=24,940)\end{array}$ & $\begin{array}{l}\text { Super-super obese } \\
(n=5723)\end{array}$ & \\
\hline & mean $\pm \mathrm{SD}$ & mean $\pm \mathrm{SD}$ & $p$ value \\
\hline Age (years) & $42.63 \pm 11.88$ & $41.65 \pm 11.22$ & $<0.0005$ \\
\hline Pre-op BMI $\left(\mathrm{kg} / \mathrm{m}^{2}\right)$ & $53.91 \pm 2.75$ & $63.67 \pm 2.7$ & $<0.0005$ \\
\hline Operation length (min) & $89.67 \pm 49.03$ & $92.65 \pm 51.09$ & $<0.0005$ \\
\hline \multirow[t]{2}{*}{ LOS (days) } & $1.76 \pm 1.3$ & $1.88 \pm 1.36$ & $<0.0005$ \\
\hline & $n(\%)$ & $n(\%)$ & $p$ value \\
\hline Sex & $18,780(75.3)$ & $4106(71.7)$ & $<0.0005$ \\
\hline Gastroesophageal reflux disease & $7380(29.6)$ & $1597(27.9)$ & 0.01 \\
\hline Limited ambulation & $594(2.4)$ & $261(4.6)$ & $<0.0005$ \\
\hline History of MI or PCI/PTCA or cardiac surgery & $822(3.3)$ & $159(2.8)$ & 0.05 \\
\hline Hypertension & $12,893(51.7)$ & $3230(56.4)$ & $<0.0005$ \\
\hline Hyperlipidemia & $5309(21.3)$ & $1159(20.3)$ & 0.08 \\
\hline Vein thrombosis requiring therapy & $504(2)$ & $165(2.9)$ & $<0.0005$ \\
\hline Venous stasis & $397(1.6)$ & $171(3.0)$ & $<0.0005$ \\
\hline End-stage renal disease/dialysis & $69(0.3)$ & $19(0.3)$ & 0.48 \\
\hline Renal insufficiency (creatinine $>2.0 \mathrm{mg} / \mathrm{dl}$ ) & $188(0.8)$ & $49(0.9)$ & 0.43 \\
\hline Therapeutic anticoagulation & $814(3.3)$ & $252(4.4)$ & $<0.0005$ \\
\hline Previous obesity surgery/foregut surgery & $326(1.3)$ & $56(1.0)$ & 0.04 \\
\hline Diabetes mellitus & $6856(27.5)$ & $1687(29.5)$ & 0.002 \\
\hline Diabetes mellitus requiring insulin & $2157(8.6)$ & $559(9.8)$ & 0.007 \\
\hline Current smoker within 1 year & $2359(9.5)$ & $539(9.4)$ & 0.93 \\
\hline Partially or fully dependent & $353(1.4)$ & $154(2.7)$ & $<0.0005$ \\
\hline Chronic obstructive pulmonary disease & $522(2.1)$ & $152(2.7)$ & 0.01 \\
\hline Oxygen dependent & $269(1.1)$ & $115(2.0)$ & $<0.0005$ \\
\hline History of pulmonary embolism & $385(1.3)$ & $140(2.4)$ & $<0.0005$ \\
\hline Obstructive sleep apnea & $11,620(46.6)$ & $3184(55.6)$ & $<0.0005$ \\
\hline Chronic steroid/immunosuppressant & $418(1.7)$ & $100(1.7)$ & 0.71 \\
\hline Pre-op IVC filter & $292(1.2)$ & $197(3.4)$ & $<0.0005$ \\
\hline ASA class 3 or 4 & $21,363(85.7)$ & $5197(90.8)$ & $<0.0005$ \\
\hline
\end{tabular}

$M I$ myocardial infarction, $P C I$ percutaneous coronary intervention, $P T C A$ percutaneous transluminal coronary angioplasty, IVC inferior vena cava, ASA American Society of Anesthesiologists compared to $12.6 \%$ of patients with SO patients experiencing a complication, $p<0.0001$. The specific 30 -day complications of unplanned intubation, wound disruption, unplanned ICU admission, re-admission, and ED visit without admission occurred at a significantly increased rate in SSO patients than SO patients (Table 2).

Among patients with SSO, patients undergoing LRYGB were more likely than patients undergoing LSG to be female ( $76 \%$ vs. $70 \%, p<0.0005)$, have GERD $(31.5 \%$ vs. $26.2 \%$, $p<0.0005)$, diabetes mellitus $(34.1 \%$ vs. $27.3 \%, p<0.0005)$, and obstructive sleep apnea $(58.6 \%$ vs. $54.3 \%, p<0.002)$ (Table 3). Among patients with SSO, patients undergoing LRYGB were more likely than patients undergoing LSG to have an unplanned intubation $(0.6 \%$ vs. $0.2 \%, p=0.01)$, transfusion within $72 \mathrm{~h}$ of surgery $(0.8 \%$ vs. $0.3 \%, p=0.02)$, unplanned ICU admission ( $1.7 \%$ vs. $0.8 \%, p=0.003)$, ED visit without re-admission ( $10.6 \%$ vs. $7.1 \%, p<0.0005)$, readmission $(6.4 \%$ vs. $4.1 \%, p<0.0005)$, intervention $(2.9 \%$ vs. $1.2 \%, p<0.0005)$, and re-operation $(2.3 \%$ vs. $0.8 \%$, $p<0.0005$ ) (Table 4).

Among the patients who underwent LSG, those with SSO had an average BMI of $63.7 \pm 2.7 \mathrm{~kg} / \mathrm{m}^{2}$ and were significantly younger $(41.8 \pm 11.3$ vs. $42.5 \pm 11.9$ years, $p<0.0001)$, had longer procedures times $(79.2 \pm 40.7$ vs. $75.7 \pm 39.6 \mathrm{~min}$, $p<0.0001)$ and longer lengths of stay than SO LSG $(1.72 \pm$ 1.1 vs. $1.64 \pm 1.2$ days, $p<0.0001)$ with an average BMI of $52.9 \pm 2.8 \mathrm{~kg} / \mathrm{m}^{2}$. SSO LSG patients had a lower percentage of female patients than SO LSG $(70 \%$ vs $74 \%, p<0.0001)$. Similar to comparing all bariatric procedures between SSO and SO, SSO LSG patients had a significantly increased rate of the same individual pre-operative co-morbidities (Supplementary Table 1). Patients with SSO who underwent 
Table 2 Occurrence of 30-day peri-operative complications of super obese (BMI $50-60 \mathrm{~kg} / \mathrm{m}^{2}$ ) compared to super-super obese patients (BMI 60.1-69.9 kg/m²) undergoing both Roux-en-Y gastric bypass and sleeve gastrectomy

\begin{tabular}{|c|c|c|c|}
\hline & $\begin{array}{l}\text { Super obese } \\
n=24,940\end{array}$ & $\begin{array}{l}\text { Super-super obese } \\
n=5723\end{array}$ & \\
\hline & $n(\%)$ & $n(\%)$ & $p$ value \\
\hline Acute renal failure & $19(0.1)$ & $6(0.1)$ & 0.49 \\
\hline Progressive renal insufficiency & $16(0.1)$ & $8(0.1)$ & 0.07 \\
\hline Superficial incisional SSI & $156(0.6)$ & $404(0.7)$ & 0.53 \\
\hline Deep incisional SSI & $18(0.1)$ & $6(0.1)$ & 0.43 \\
\hline Organ space SSI & $44(0.2)$ & $11(0.2)$ & 0.80 \\
\hline Wound disruption & $15(0.1)$ & $8(0.1)$ & 0.05 \\
\hline Post-op sepsis & $22(0.1)$ & $5(0.1)$ & 0.98 \\
\hline Post-op septic shock & $13(0.1)$ & $4(0.1)$ & 0.61 \\
\hline Clostridium difficile colitis & $34(0.1)$ & $8(0.1)$ & 0.95 \\
\hline Urinary tract infection & $117(0.5)$ & $33(0.6)$ & 0.29 \\
\hline Peripheral nerve injury & $4(0.0)$ & $0(0.0)$ & 0.34 \\
\hline Transfusion within $72 \mathrm{~h}$ of surgery & $141(0.6)$ & $27(0.5)$ & 0.39 \\
\hline Myocardial infarction & $7(0.0)$ & $0(0.0)$ & 0.21 \\
\hline Cardiac arrest requiring $\mathrm{CPR}$ & $11(0.0)$ & $2(0.0)$ & 0.76 \\
\hline Stroke/cerebrovascular accident & $2(0.0)$ & $2(0.0)$ & 0.11 \\
\hline Pneumonia & $64(0.3)$ & $11(0.2)$ & 0.37 \\
\hline Ventilator $>48 \mathrm{~h}$ & $17(0.1)$ & $9(0.2)$ & 0.04 \\
\hline Unplanned intubation & $45(0.2)$ & $19(0.3)$ & 0.02 \\
\hline Unplanned ICU admission & $185(0.7)$ & $63(1.1)$ & 0.006 \\
\hline $\begin{array}{l}\text { Initiation of anticoagulation for vein } \\
\text { thrombosis/pulmonary embolism }\end{array}$ & $115(0.5)$ & $31(0.5)$ & 0.43 \\
\hline ED visit without admission & $1728(6.9)$ & $470(8.2)$ & 0.001 \\
\hline Re-admission & $1038(4.2)$ & $276(4.8)$ & 0.03 \\
\hline Intervention & $357(1.4)$ & $100(1.7)$ & 0.08 \\
\hline Re-operation & $280(1.1)$ & $74(1.3)$ & 0.28 \\
\hline Mortality & $33(0.1)$ & $10(0.2)$ & 0.44 \\
\hline
\end{tabular}

SSI surgical site infection, $C P R$ cardiopulmonary resuscitation, $I C U$ intensive care unit, $E D$ emergency department
LSG were more likely to experience a complication compared to patients with SO $(13.0 \%$ vs. $10.6 \%, p<0.0001)$, but the only individual categories with a statistically significant increase in complications were wound disruption $(0.2 \%$ vs. $0.1 \%, p=0.03)$, re-admission ( $4.1 \%$ vs. $3.2 \%, p=0.003$ ), and ED visits without admission ( $7.1 \%$ vs. $6.1 \%, p=0.02)$ (Supplementary Table 3).

Among the patients who underwent LRYGB, those with SSO were younger $(41.5 \pm 11.0$ vs. $42.8 \pm 11.7$ years, $p<0.0001)$ and a lower percentage of female patients than SO RYGB patients $(75.5 \%$ vs $78.1 \%, p=0.02)$. SSO LRYGB patients had longer lengths of stay than SO patients undergoing LRYGB $(2.22 \pm 1.8$ vs. $2.02 \pm 1.4$ days, $p<0.0001)$. The average BMI of SSO LRYGB was $63.7 \pm$ $2.7 \mathrm{~kg} / \mathrm{m}^{2}$ and SO LRYGB was $54.0 \pm 2.8 \mathrm{~kg} / \mathrm{m}^{2}, p<0.0001$. There was not a significant difference in the length of procedure for patients with SSO undergoing LRYGB $(121.6 \pm 58.5$ vs. $120.2 \pm 53.6 \mathrm{~min}, p=0.34)$ compared to $\mathrm{SO}$ patients.
However, there was no statistical difference detected between SSO and SO LRYGB patients in the percent with a history of $\mathrm{MI} /$ coronary intervention/cardiac surgery, hyperlipidemia, history of previous obesity/foregut surgery, or chronic obstructive pulmonary disease (Supplementary Table 2). Patients with SSO who underwent LRYGB were more likely to experience a complication compared to patients with $\mathrm{SO}$ ( $20.1 \%$ vs. $16.9 \%, p=0.001)$ with statistically significant increased risk of the individual complications of prolonged ventilation $(0.4 \%$ vs. $0.1 \%, p=0.003)$, unplanned intubation $(0.6 \%$ vs. $0.3 \%, p=0.03)$, post-operative urinary tract infection $(1.0 \%$ vs. $0.5 \%, p=0.02)$, unplanned ICU admission (1.7\% vs. $0.9 \%, p=0.004)$, and ED visits without admission $(10.6 \%$ vs. $8.6 \%, p=0.01)$ (Supplementary Table 4$)$.

For the outcome of unplanned intubation, $\mathrm{SSO}$, which is significant on univariate analysis (OR 1.84, $p=0.03$ ), is no longer significant but HTN, age limited ambulation, OSA, and BMI are. For wound disruption, only SSO was significant on 
Table 3 Demographics and presurgical co-morbidities of supersuper obese patients (BMI 60.1$69.9 \mathrm{~kg} / \mathrm{m}^{2}$ ) undergoing laparoscopic Roux-en-Y gastric bypass versus sleeve gastrectomy

\begin{tabular}{|c|c|c|c|}
\hline & RYGB $(n=1816)$ & $\operatorname{LSG}(n=3907)$ & \\
\hline & mean $\pm \mathrm{SD}$ & mean $\pm \mathrm{SD}$ & $p$ value \\
\hline Age (years) & $41.49 \pm 11.03$ & $41.73 \pm 11.31$ & 0.29 \\
\hline Pre-op BMI $\left(\mathrm{kg} / \mathrm{m}^{2}\right)$ & $63.72 \pm 2.71$ & $63.65 \pm 2.70$ & 0.73 \\
\hline Operation length (min) & $121.64 \pm 58.52$ & $79.18 \pm 40.74$ & $<0.0005$ \\
\hline \multirow[t]{2}{*}{ LOS (days) } & $2.22 \pm 1.70$ & $1.74 \pm 1.17$ & $<0.0005$ \\
\hline & $n(\%)$ & $n(\%)$ & $p$ value \\
\hline Sex & $1371(75.5)$ & $2735(70)$ & $<0.0005$ \\
\hline Gastroesophageal reflux disease & $572(31.5)$ & $1025(26.2)$ & $<0.0005$ \\
\hline Limited ambulation & $83(4.6)$ & $178(4.6)$ & 0.98 \\
\hline History of MI or PCI/PTCA or cardiac surgery & $50(2.8)$ & $109(2.8)$ & 0.94 \\
\hline Hypertension & $1051(57.9)$ & $2179(55.8)$ & 0.14 \\
\hline Hyperlipidemia & $396(34.2)$ & $763(19.5)$ & 0.05 \\
\hline Vein thrombosis requiring therapy & $59(3.2)$ & $106(2.7)$ & 0.26 \\
\hline Venous stasis & $47(2.6)$ & $124(3.2)$ & 0.23 \\
\hline End-stage renal disease/dialysis & $4(0.2)$ & $15(0.4)$ & 0.32 \\
\hline Renal insufficiency (creatinine $>2.0 \mathrm{mg} / \mathrm{dl}$ ) & $18(1.0)$ & $31(0.8)$ & 0.45 \\
\hline Therapeutic anticoagulation & $338(3.5)$ & $728(3.5)$ & 0.84 \\
\hline Previous obesity surgery/foregut surgery & $17(0.9)$ & $39(1.0)$ & 0.82 \\
\hline Diabetes mellitus & $620(34.1)$ & $1067(27.3)$ & $<0.0005$ \\
\hline Diabetes mellitus requiring insulin & $1122(11.6)$ & $1594(7.6)$ & $<0.0005$ \\
\hline Current smoker within 1 year & $158(8.7)$ & $381(9.8)$ & 0.21 \\
\hline Partially or fully dependent & $46(2.5)$ & $108(2.8)$ & 0.62 \\
\hline Chronic obstructive pulmonary disease & $48(2.6)$ & $104(2.7)$ & 0.97 \\
\hline Oxygen dependent & $49(2.7)$ & $66(1.7)$ & 0.01 \\
\hline History of pulmonary embolism & $43(2.4)$ & $97(2.5)$ & 0.79 \\
\hline Obstructive sleep apnea & $1064(58.6)$ & $2120(54.3)$ & 0.002 \\
\hline Chronic steroid/immunosuppressant & $31(1.7)$ & $69(1.8)$ & 0.87 \\
\hline Pre-op IVC filter & $60(3.3)$ & $137(3.5)$ & 0.70 \\
\hline ASA class 3 or 4 & $1694(93.3)$ & $3503(89.7)$ & $<0.0005$ \\
\hline
\end{tabular}

$M I$ myocardial infarction, $P C I$ percutaneous coronary intervention, PTCA percutaneous transluminal coronary angioplasty, $I V C$ inferior vena cava, $A S A$ American Society of Anesthesiologists univariate modeling (OR 2.30, $p=0.05$ ), with no significant association with sex, age, diabetes mellitus, limited ambulation, hypertension, and sleep apnea. For ED visits, not requiring re-admission, SSO (OR 1.2, $p<0.0005$ ), sex (OR 1.73, $p<0.0005)$, and age (OR $0.98, p<0.0005)$ were significant on multivariate regression. For re-admission, BMI (OR 1.01, $p=0.02)$, sex (OR 1.30, $p<0.0005)$, HTN (OR 1.20, $p=$ $0.006)$, and limited ambulation $(\mathrm{OR}=1.69, p<0.0005)$ were significantly associated on multivariate regression. For each complication, except unplanned intubation, either obesity status or BMI is significant on multivariate logistic regression modeling, which supports our conclusions that patients with SSO have more complications regardless of pre-operative comorbidities.

On univariate analysis, BMI did not predict the increased rate of prolonged ventilation or the need for reintubation, but did predict the increased risk of ICU admissions (OR 1.49, CI 1.12-1.99, $p=0.007$ ). On multivariable analysis, controlling for obesity class, the risk for re-intubation increased 6.25 -fold for patients that had COPD (CI 2.92-13.16, $p<0.0005$ ) and 4.65-fold for patients with impaired ambulation (CI 2.20-9.80, $p<0.0005$ ). Controlling for obesity class, the risk for unplanned ICU admission increased 4.83-fold for patients with COPD (CI 3.41-7.41, $p<0.0005$ ), 2.49-fold for obstructive sleep apnea (CI 1.89-3.27, $p<0.0005$ ), and 3.47fold for impaired ambulation (CI 2.42-5.38, $p<0.0005$ ).

As shown in Fig. 1, when compared to SO patients undergoing LRYGB, the relative risk (RR) of a 30-day complication significantly decreased with both SO LSG $(\mathrm{RR}=0.62$, CI $0.59-0.67, p<0.0001)$ and SSO LSG $(0.76$, CI $0.70-0.84$, $p<0.0001)$. The relative risk increase for a 30 -day complication for a patient with SSO undergoing a LRYGB compared to SO LRYGB was 1.19 (CI 1.07-1.32, $p<0.0001$ ). 
Table 4 Occurrence of 30-day peri-operative complications of super-super obese patients (BMI $\left.60.1-69.9 \mathrm{~kg} / \mathrm{m}^{2}\right)$ undergoing laparoscopic Roux-en-Y gastric bypass versus sleeve gastrectomy

\begin{tabular}{|c|c|c|c|}
\hline & $\begin{array}{l}\text { RYGB } \\
(n=1816)\end{array}$ & $\begin{array}{l}\text { LSG } \\
(n=3907)\end{array}$ & \\
\hline & $n(\%)$ & $n(\%)$ & $p$ value \\
\hline Acute renal failure & $3(0.2)$ & $3(0.1)$ & 0.34 \\
\hline Progressive renal insufficiency & $3(0.2)$ & $5(0.1)$ & 0.73 \\
\hline Superficial incisional SSI & $0(0.0)$ & $0(0.0)$ & - \\
\hline Deep incisional SSI & $1(0.1)$ & $0(0.0)$ & 0.14 \\
\hline Organ space SSI & $1(0.1)$ & $0(0.0)$ & 0.14 \\
\hline Wound disruption & $1(0.1)$ & $7(0.2)$ & 0.24 \\
\hline Post-op sepsis & $0(0.0)$ & $0(0.0)$ & - \\
\hline Post-op septic shock & $0(0.0)$ & $0(0.0)$ & - \\
\hline Clostridium difficile colitis & $4(0.2)$ & $4(0.1)$ & 0.27 \\
\hline Urinary tract infection & $1(0.1)$ & $1(0.0)$ & 0.58 \\
\hline Peripheral nerve injury & $0(0.0)$ & $0(0.0)$ & - \\
\hline Transfusion within $72 \mathrm{~h}$ of surgery & $14(0.8)$ & $13(0.3)$ & 0.02 \\
\hline Myocardial infarction & $0(0.0)$ & $0(0.0)$ & - \\
\hline Cardiac arrest requiring $\mathrm{CPR}$ & $1(0.1)$ & $1(0.0)$ & 0.58 \\
\hline Stroke/cerebrovascular accident & $0(0.0)$ & $2(0.1)$ & 0.34 \\
\hline Pneumonia & $0(0.0)$ & $0(0.0)$ & - \\
\hline Ventilator $>48 \mathrm{~h}$ & $0(0.0)$ & $1(0.0)$ & 0.50 \\
\hline Unplanned intubation & $11(0.6)$ & $8(0.2)$ & 0.01 \\
\hline Unplanned ICU admission & $31(1.7)$ & $32(0.8)$ & 0.003 \\
\hline $\begin{array}{l}\text { Initiation of anticoagulation for vein } \\
\text { thrombosis/pulmonary embolism }\end{array}$ & $13(0.7)$ & $18(0.5)$ & 0.22 \\
\hline ED visit without admission & $192(10.6)$ & $278(7.1)$ & $<0.0005$ \\
\hline Re-admission & $116(6.4)$ & $160(4.1)$ & $<0.0005$ \\
\hline Intervention & $53(2.9)$ & $47(1.2)$ & $<0.0005$ \\
\hline Re-operation & $41(2.3)$ & $33(0.8)$ & $<0.0005$ \\
\hline Mortality & $3(0.2)$ & $7(0.2)$ & 0.91 \\
\hline
\end{tabular}

SSI surgical site infection, $C P R$ cardiopulmonary resuscitation, $I C U$ intensive care unit, $E D$ emergency department

\section{Discussion}

Our study demonstrates a significantly higher complication rate for SSO patients undergoing LRYGB compared to LSG as well as a significant increase in complications in patients with SSO compared to SO. This was coupled with an increase in pre-operative co-morbidities of SSO compared to SO patients. SSO patients had an almost 2-fold increase in severe co-morbidities including limited ambulation, lack of functional independence, oxygen dependence, history of a PE, and history of a pre-op IVC filter. Considering the increase comorbidity status of SSO patients, SSO LSG still had a significantly lower relative risk of a complication compared to LRYGB, even for SO patients.

We found a high overall complication rate $(13.1 \%)$ in this study compared to other large bariatric database studies ranging from 6.3 to $14.8 \%[8,9]$. However, our study only selected for patients with SO and SSO compared to the BOLD (average BMI $46.5 \mathrm{~kg} / \mathrm{m}^{2}$, laparoscopic and open RYGB), NSQIP (average BMI $44.1 \mathrm{~kg} / \mathrm{m}^{2}$, LRYGB only), and Michigan Bariatric Surgical Collaborative (average BMI $45.7 \mathrm{~kg} / \mathrm{m}^{2}$, LRYGB, and LSG) studies [3, 8, 9]. Comparing patients in our study to the BOLD, NSQIP, and Michigan Bariatric Surgical Collaborate, there were similar rates of hypertension (52.6\% vs. $52.1 \%, 53.6 \%$, and $53.6 \%$ respectively) and diabetes mellitus ( $27.9 \%$ vs. $25.2 \%, 27.3 \%$, and $35 \%$ respectively) but with higher rates of sleep apnea $48.3 \%$ compared to the BOLD database (35.7\%). Individually, the rates of specific complications compared favorably with NSQIP and the Michigan Bariatric Surgical Collaborative with similar rates of acute renal failure ( $0.1 \%$ vs. $0.1 \%$ and $0.2 \%$ respectively), urinary tract infection $(0.5 \%$ vs. $0.7 \%$ and $0.3 \%$ respectively), pneumonia ( $0.2 \%$ vs. $0.5 \%$ and $0.9 \%$ respectively), unplanned intubation $(0.2 \%$ vs. $0.3 \%$ and $0.4 \%$ respectively), vein thrombosis ( $0.1 \%$ vs. $0.3 \%$ and $0.4 \%$ respectively), and death ( $0.1 \%$ vs. $0.2 \%$ and $0.1 \%$ respectively). Overall, the higher complication rate in this study appears to be driven by the increased number of complications recorded in the 2016 
Fig. 1 Relative risk and 95\% confidence interval of a complication by bariatric procedure and BMI category. Error bars show $95 \%$ confidence intervals. Super obese laparoscopic Roux-en-Y gastric bypass (SO LRYGB) was used as the reference group. ${ }^{*} p<0.0001$. LSG laparoscopic sleeve gastrectomy, SSO super-super obese

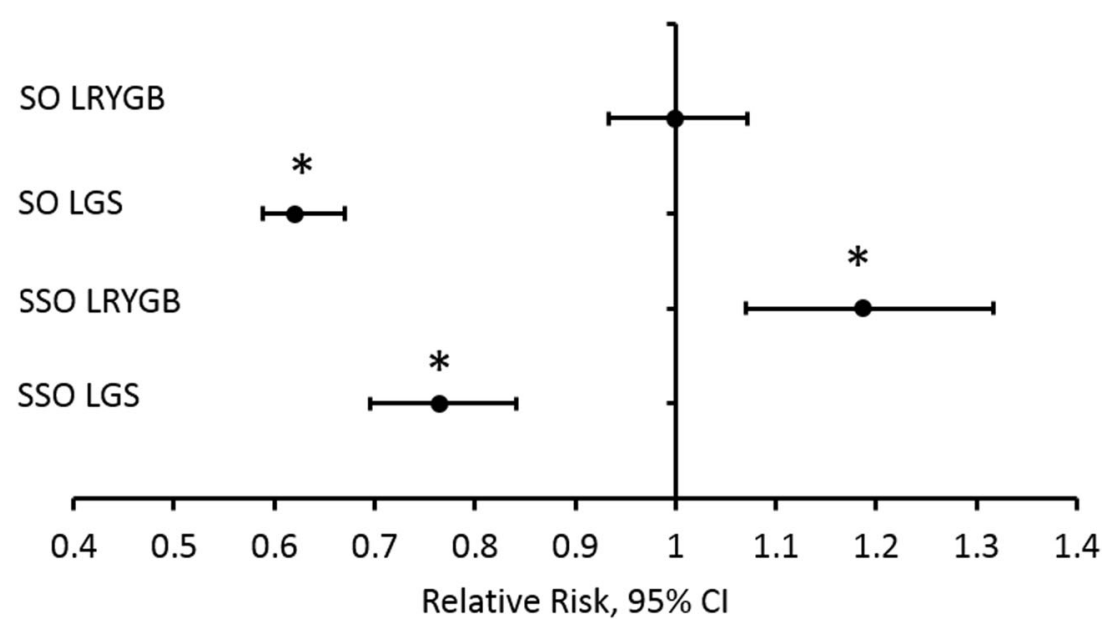

MBSAQIP data registry, as it captures several complications not recorded in BOLD, NSQIP, and the Michigan Bariatric Surgical Collaborative data including prolonged ventilation, peripheral nerve injury, unplanned intubation, unplanned ICU admission, clostridium colitis, re-admission, re-operation, and ED visits with ED visits being the most common complication for all patients $(7.2 \%)$.

A recent prospective study (485 patients, 2010-2017, average BMI $48.2 \mathrm{~kg} / \mathrm{m}^{2}$ ) did not show any significant difference in complications, re-admissions, or re-operations within 30 days between patients undergoing LRYGB or LSG [10]. And although a large meta-analysis of singlecenter studies did not find any difference in leak or mortality rates between LRYGB and LSG, most reported data shows an increased complication rate in patients undergoing LRYGB versus LSG [11-13]. In the Michigan Bariatric Surgery Collaborative, the percentage of patients having a severe complication was of 3.3\% for LRYGB compared to $2.3 \%$ for LSG [8]. Analysis of the BOLD registry found $14.9 \%$ of patients experiencing one or more complications for LRYGB compared to $10.8 \%$ for LSG [9]. These large database studies are consistent with our finding of complication rates of $16.9 \%$ for SO and $20.1 \%$ for SSO patients undergoing LRYGB compared to $10.6 \%$ for $\mathrm{SO}$ and $13.0 \%$ for SSO patients undergoing LSG.

Our data show a clear and significant increase in complications in patients with SSO compared to SO, regardless of procedure type. This is consistent with previous US database analysis from 2005 to 2009 reporting an increased risk of complications for patients with a BMI $>60 \mathrm{~kg} / \mathrm{m}^{2}$ undergoing bariatric surgery $[3,5,6]$. The more recent Scandinavian Obesity Surgery Registry (SOSR) database did not find pre-operative BMI to be predictive of post-operative complications; however, the SOSR database contained fewer than $1 \%$ patients with SSO [7]. Similarly, a single-center retrospective review in Germany between 2010 and 2013, did not see increased complications in the SSO but acknowledged that patients with SSO were most commonly operated on by the highest volume and most experienced surgeons potentially biasing their results [4].

Our study is subject to limitations and may not be generalizable to other groups or locations. The MBSAQIP database comes from accredited bariatric centers. The values reported might not apply to LRYGBs or LSGs being performed at non-MBSAQIP accredited centers. Additionally, though data is entered by a certified metabolic and bariatric clinical reviewer, the data can still contain coding errors and omissions. Our study is most limited by the difficulties in separating BMI as an independent predictor of complications from the significantly increased pre-operative morbidity of SSO patients. Additionally, the large size of the dataset means that it is relatively easy to generate statistically significant results which are not clinically meaningful. For example, the statistically significant difference in operative time and length of stay for SSO versus SO patients undergoing LSG is 3 min and 0.08 days which is not clinically significant. For the most common complication of ED visits without an admission, OSA, HTN, COPD, and limited ambulation pre-operatively were not significant predictors on multivariate analysis. However, for the complication of re-intubation, COPD and ambulatory status were both significant risk factors. Similarly, COPD, obstructive sleep apnea, and ambulatory status were significant risk factors for ICU admission post-op. The dataset accessed for this study did not include weight loss and co-morbidity resolution and is a critical factor when considering the risk compared to benefit of these operations and adequate procedure selection for the individual patient. It is quite possible that the increased peri-operative risk of SSO patients with LRYGB may be mitigated if long-term outcomes reveal superior co-morbidity improvement compared to LSG and thus long-term cardiovascular and mortality risk reduction [14]. 


\section{Conclusions}

Patients with SSO undergoing LRYGB or LSG have an increased risk of post-operative 30-day complications compared to patients with SO. Patients with both SO and SSO who undergo LSG have a significantly decreased relative risk of complications compared to both SO RYGB and SSO RYGB patients. For patients with SSO, LSG may be the preferred procedure of choice to counter the increased peri-operative risk associated with multiple pre-operative co-morbidities affording a lower 30-day post-operative complication profile compared to LRYGB.

\section{Compliance with Ethical Standards}

The authors declare that they have no conflict of interest. This article does not contain any studies with human participants or animals performed by any of the authors. For this type of study, formal consent is not required and does not apply.

\section{References}

1. Centers for Disease Control and Prevention; National Center for Health Statistics. National Health and Nutrition Examination Survey. https://www.cdc.gov/obesity/data/adult.html. Accessibility verified October 20, 2018.

2. Sturm R, Hattori A. Morbid obesity rates continue to rise rapidly in the United States. Int J Obes. 2013;37(6):889-91.

3. Maciejewski ML, Winegar DA, Farley JF, et al. Risk stratification of serious adverse events after gastric bypass in the Bariatric Outcomes Longitudinal Database. Surg Obes Relat Dis. 2012;8(6):671-7.

4. Duprée A, El gammal AT, Wolter S, et al. Perioperative short-term outcome in super-super-obese patients undergoing bariatric surgery. Obes Surg. 2018;28(7):1895-901.
5. Gupta PK, Franck C, Miller WJ, et al. Development and validation of a bariatric surgery morbidity risk calculator using the prospective, multicenter NSQIP dataset. J Am Coll Surg. 2011;212(3):301-9.

6. Turner PL, Saager L, Dalton J, et al. A nomogram for predicting surgical complications in bariatric surgery patients. Obes Surg. 2011;21(5):655-62.

7. Stenberg E, Szabo E, Agren G, et al. Early complications after laparoscopic gastric bypass surgery: results from the Scandinavian Obesity Surgery Registry. Ann Surg. 2014;260(6):1040-7.

8. Finks JF, Kole KL, Yenumula PR, et al. Predicting risk for serious complications with bariatric surgery: results from the Michigan Bariatric Surgery Collaborative. Ann Surg. 2011;254(4):633-40.

9. Demaria EJ, Pate V, Warthen M, et al. Baseline data from American society for metabolic and bariatric surgery-designated bariatric surgery centers of excellence using the bariatric outcomes longitudinal database. Surg Obes Relat Dis. 2010;6(4):347-55.

10. Lynn W, Ilczyszyn A, Rasheed S, et al. Laparoscopic Roux-en-Y gastric bypass is as safe as laparoscopic sleeve gastrectomy. Results of a comparative cohort study. Ann Med Surg (Lond). 2018;35:3843.

11. Zellmer JD, Mathiason MA, Kallies KJ, et al. Is laparoscopic sleeve gastrectomy a lower risk bariatric procedure compared with laparoscopic Roux-en-Y gastric bypass? A meta-analysis. Am J Surg. 2014;208(6):903-10.

12. Janik MR, Rogula TG, Mustafa RR, et al. Safety of revision sleeve gastrectomy compared to Roux-Y gastric bypass after failed gastric banding: analysis of the MBSAQIP. Ann Surg. 2019;269(2):299303.

13. Janik MR, Mustafa RR, Rogula TG, et al. Safety of laparoscopic sleeve gastrectomy and Roux-en-Y gastric bypass in elderly patients - analysis of the MBSAQIP. Surg Obes Relat Dis. 2018;14(9):1276-82.

14. Golzarand M, Toolabi K, Farid R. The bariatric surgery and weight losing: a meta-analysis in the long- and very long-term effects of laparoscopic adjustable gastric banding, laparoscopic Roux-en-Y gastric bypass and laparoscopic sleeve gastrectomy on weight loss in adults. Surg Endosc. 2017;31:4331-45.

Publisher's Note Springer Nature remains neutral with regard to jurisdictional claims in published maps and institutional affiliations. 